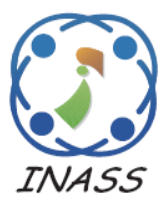

\title{
SOC Estimation for Traction Battery Pack of Electric Vehicles with Battery Degradation Tracking
}

\author{
Waiard Saikong ${ }^{1}$ \\ Chaiyut Sumpavakup ${ }^{2}$ \\ Thanatchai Kulworawanichpong ${ }^{1 *}$ \\ ${ }^{1}$ School of Electrical Engineering, Suranaree University of Technology, \\ Nakhon Ratchasima 30000, Thailand \\ ${ }^{2}$ Research Centre for Combustion Technology and Alternative Energy - CTAE \\ College of Industrial Technology, King Mongkut's University of Technology North Bangkok, \\ Bangkok 10800, Thailand \\ * Corresponding author’s Email: thanatchai@gmail.com
}

\begin{abstract}
In the electric vehicle industry, a good estimation of a traction battery pack or the state of charge (SOC) is crucial as it reflects how far a vehicle travel before recharging. As the battery degrades, its behavior and the associated parameters such as internal resistance, capacity and SOC-OCV (open circuit voltage) mapping changes. Thus, a battery model has to take into account the changes in the battery parameters for it to be accurate throughout the battery lifetime. For such a model to be computational intensive, it requires powerful processors. With limited calculation performance processors found in vehicles, the model fidelity is normally compromised. In this paper, two battery models are used to accurately estimate traction battery SOC; The Ohmic resistance model is used to sense changes in battery internal resistance, when the change is significant, the resistor-capacitor (RC) model is used to update the battery SOC-OCV curve which is used to estimate the battery initial SOC. Hence, the coulomb counting method is used to update the battery SOC. The real operational battery data from PEA Ze-Bus (Zero-Emission bus of the Provincial Electricity Authority of Thailand) are used in this study. The proposed algorithm used to test the state of charge of the battery has been verified and illustrates the error of SOC estimation at $3.31 \%$, less than the unadaptable model.
\end{abstract}

Keywords: Electric vehicle, Traction battery pack modelling, Battery parameter estimation, Online parameter tracking, SOC estimation algorithm.

\section{Introduction}

Nowadays, electric vehicles are gaining in popularity due to environmental and fuel security concerns [1-4]. Lithium-ion based battery is currently an energy storage device that features properties to meet requirements for electric vehicles, specifically, high energy and power densities, high coulombic efficiency, and low cycle cost. To describe the behavior of a traction battery, a variety of battery models have been proposed in the field of research in the past decade [5-10]. For equivalent circuit battery model, as the model becomes more complex (more components added into it) the fidelity of the model tends to increase, this can be appropriate for simulation works. However, when the model has to be implemented to an embedded system for practical use, a challenge usually arises due to limited calculation performance of the hardware [11]. Thus, for a battery model to be implemented on limited performance calculation hardware for real time parameter estimation, its fidelity is always compromised.

A battery model that is accurate and reliable throughout the battery lifetime is very important in electric vehicles (EVs), to accurately estimate and update battery parameters responding to a unique EV intermittent load, a function of route and driving profiles. Electric vehicles users may find themselves stranded along the road just because the estimated battery state of charge (SOC) was far above the actual SOC. Generally, manufacturers acquire parameters of a battery cell by charge and discharge impulse 
testing, cell capacity is acquired by various levels of constant current discharging under given temperature [6].

Despite a practical EV load being far from the constant current, battery cells are connected in series and/or parallel depending on the application and controlled by a battery management system (BMS). Parameters of an entire EV battery system may not be accurately reflected by a single battery cell. Moreover, when a battery has been cycled a number of times over the course, its behavior and consequently its parameters will change. Obviously then, it is necessary to update or recalculate the battery parameters throughout its lifetime, in order to accurately estimate a crucial parameter or the SOC. Battery SOC reflects how much energy is in the battery and consequently how far a vehicle can go before recharging [12].

Battery SOC cannot be measured directly, the well-known methods in current research used to estimate SOC are the coulomb counting technique and SOC-OCV correlation (the hysteresis curve), which is normally scaled up from a battery cell level. Battery pack SOC depends on various factors such as self-discharge, charge and discharge current rate, number of charging cycle and the BMS parameter configuration $[13,14]$. In this paper a novel SOC estimation method is proposed using only two measured parameters, current and voltage. The proposed method uses two battery equivalent circuits, the ohmic resistance equivalent circuit and resistorcapacitor (RC) equivalent circuit. Using the battery current and terminal voltage, the ohmic resistance equivalent circuit model estimates battery internal resistance and tracks its changes, once the change in estimated, internal resistance will then be significant, a request is sent to the $\mathrm{RC}$ equivalent circuit to update the SOC-OCV curve. The up to date SOC-OCV curve is used to estimate battery's initial SOC. Data from PEA Ze-Bus (Zero-Emission bus of the Provincial Electricity Authority of Thailand) are applied in this study. Prior to the selection of using the RC model to update SOC-OCV curve, a comparison between ohmic resistance model and RC model are done to establish the fidelity of the RC model.

The rest of the paper is organized as followed, the battery parameter estimation is presented in Section 2. Section 3 presents specifications of the test vehicle, field data, and proposed algorithm. The results and discussion are then presented in Section 4. Finally, the conclusion is presented in Section 5.

\section{Battery parameter estimation}

\subsection{Ohmic resistance battery model}

The ohmic resistance battery model [14] as shown in Fig. 1, can be used to roughly estimate the battery's internal resistance $\left(R_{o}\right)$ and open circuit voltage $(\mathrm{OCV})$ given terminal voltage $\left(V_{T}\right)$ and terminal current $\left(I_{T}\right)$ which can be directly measured. The parameters for using in battery modelling are shown in Table 1.

From the equivalent circuit in Fig. 1, Eqs. (1) - (3) are developed.

$$
V_{O C}-I_{T} R_{0}=V_{T}
$$

From a system of linear equations,

$$
A_{k} \vec{x}_{k}=b_{k}
$$

\begin{tabular}{|c|c|}
\hline Notation & Definition \\
\hline$V_{O C}$ & Open circuit voltage \\
\hline$R_{0}$ & Internal resistance \\
\hline$V_{T}$ & Terminal voltage \\
\hline$I_{T}$ & Terminal current \\
\hline$A$ & State matrix (for eq.7) \\
\hline$B$ & Input matrix (for eq.7) \\
\hline$C$ & Output matrix (for eq.8) \\
\hline$D$ & Feedforward matrix (for eq.8) \\
\hline$O C V(S O C)$ & $\begin{array}{l}\text { Open circuit voltage as a function of } \\
\text { state of charge }\end{array}$ \\
\hline$C_{1}$ & Capacitance of RC model \\
\hline$R_{1}$ & Resistance of RC model \\
\hline$k$ & $\begin{array}{l}\text { The experimental data are collected up } \\
\text { to time } k\end{array}$ \\
\hline$N, n$ & Natural number \\
\hline$T$ & Sampling period \\
\hline$I$ & Identity matrix \\
\hline$S O C_{C U R V E}$ & $\begin{array}{l}\text { SOC is estimated by the SOC-OCV } \\
\text { curve }\end{array}$ \\
\hline$S O C_{A h}$ & $\begin{array}{l}\text { SOC is estimated by the coulomb } \\
\text { counting technique }\end{array}$ \\
\hline$A_{d}$ & State matrix in discretized form \\
\hline$B_{d}$ & Input matrix in discretized form \\
\hline
\end{tabular}

Table 1. Battery modelling parameters

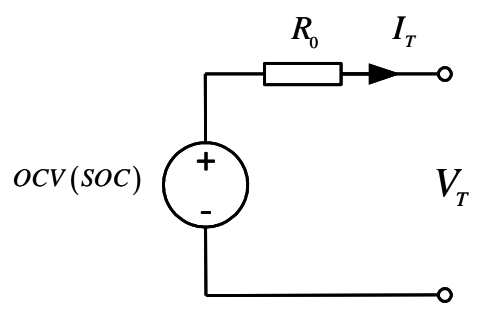

Figure.1 Battery ohmic resistance model 
Eq. (1) can be written in the form of Eq. (2)

$$
\text { then, } \quad\left[\begin{array}{ll}
1 & -I_{T, k}
\end{array}\right]\left[\begin{array}{c}
V_{O C} \\
R_{0}
\end{array}\right]=V_{T, k}
$$

Open circuit voltage $\left(V_{O C}\right)$ and $R_{O}$ are then estimated by recursive least-square estimation (RLSE). Since the Eq. (3) is not computational intensive, $V_{O C}$ and $R_{0}$ estimation can be done in realtime. The RLSE algorithm adds a new correction term and recalculates $V_{O C}$ and $R_{O}$ based on new data. Consequently, this algorithm is appropriate to track changes in parameters over a time application condition. The load current $\left(I_{T, k}\right)$ and the battery terminal voltage $\left(V_{T, k}\right)$ are acquired from real-time measurement. Eq. (2) is then modified as shown in Eq. (4) when the new data arrives.

$$
A_{k+1} \vec{x}_{k+1} \approx b_{k+1},
$$

where,

$$
\begin{aligned}
& A_{k+1}=\left[\begin{array}{cc}
1 & -I_{T, k} \\
1 & -I_{T, k+1}
\end{array}\right]=\left[\begin{array}{c}
A_{k} \\
a_{k+1}^{T}
\end{array}\right], \\
& \vec{x}_{k+1}=\left[\begin{array}{l}
V_{O C, k+1} \\
R_{0, k+1}
\end{array}\right], \quad b_{k+1}=\left[\begin{array}{c}
V_{T, k} \\
V_{T, k+1}
\end{array}\right]
\end{aligned}
$$

the least-squares error is applied to the new data as presented in Eq. (5).

$$
\vec{x}_{k+1}=\left[\begin{array}{ll}
A_{k+1}^{T} & A_{k+1}
\end{array}\right]^{-1} A_{k+1}^{T} b_{k+1},
$$

with, $\quad G_{k+1}=\left[\begin{array}{ll}A_{k+1}^{T} & A_{k+1}\end{array}\right]^{-1}$

Which uses the recursive form from the reference that can be written as shown in Eq. (6) [15-16].

$$
\vec{x}_{k+1}=\vec{x}_{k}+G_{k+1} a_{k+1}\left(V_{T, k+1}-a_{k+1}^{T} \vec{x}_{k}\right),
$$

\subsection{RC circuit model}

The resistor-capacitor (R-C) battery model $[6,17]$ shown in Fig. 2 adds a resistor in parallel with a capacitor to the ohmic model in Fig. 1 to capture the polarization effect of lithium-ion battery [18].

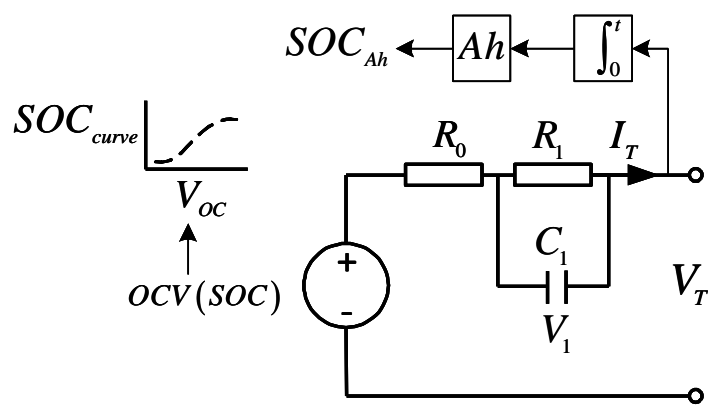

Figure.2 RC equivalent circuit model
From Fig. 2, state space equation can be written as follows.

$$
\begin{aligned}
& \dot{x}=A x+B I_{T}, \\
& V=C x+D I_{T},
\end{aligned}
$$

where, $\quad \dot{V}_{1}=\frac{-V_{1}}{C_{1} R_{1}}+\frac{I_{T}}{C_{1}}, \quad V=-I_{T} R_{0}-V_{1}$,

and $\quad V=V_{T}-V_{O C}$

The discretization formula is applied to the continuous-time state Eqs. (7) and (8) with the sampling period $\mathrm{T}$ to decrease the calculation time [15] when using offline. The SQP (sequential quadratic programming) heuristic process algorithm, can be illustrated according to the following Eq. (9). The state of charge is calculated based on the current integration as described in Fig. 2.

$$
x[n+1]=A_{d} x[n]+B_{d} I_{T}[n]
$$

where the discretized matrices are

$$
\begin{aligned}
& A_{d}=I+A T \Psi \\
& B_{d}=\Psi T B
\end{aligned}
$$

where,

$$
\begin{aligned}
\Psi \cong & I+\frac{-T}{2 R_{1} C_{1}} \times \ldots \\
& \left\{I+\frac{-T}{3 R_{1} C_{1}}\left\{I+\cdots+\frac{-T}{(N-1) R_{1} C_{1}}\left(I+\frac{-T}{N R_{1} C_{1}}\right)\right\} \ldots\right\}
\end{aligned}
$$

for $N>1$, where $N$ is a natural number

\section{Test vehicle, field data and proposed method}

To accurately estimate the battery SOC, a battery model may be improved and corrected by adding more parameters [2,7,19-20]. Adding more parameters increases the complexity of the model. On the other hand, the battery modelling using battery pack data are more accurate than those using cell data, as the battery pack includes electrical topology and BMS effects. Using BMS, the SOC always depends on the weakest cell in the battery system.

\subsection{Test vehicle}

A provincial electricity authority electric zero 
Table 2. PEA-Zebus Technical Data

\begin{tabular}{|c|c|}
\hline Specific data & Information \\
\hline \multicolumn{2}{|l|}{ Size and weight } \\
\hline Dimension $(\mathrm{w} \times 1 \times \mathrm{h})(\mathrm{m})$ & $2.55 \times 12 \times 2.96$ \\
\hline Tare mass (kg) & 12,400 \\
\hline \multicolumn{2}{|l|}{ Traction motor } \\
\hline Motor technology & $\begin{array}{l}2 \text { asynchronous hub } \\
\text { motors, } 3 \text { phases }\end{array}$ \\
\hline Rated voltage (V) & 650 \\
\hline Motor power $(\mathrm{kW})$ & $\begin{array}{l}2 \times 125 \\
2 \times 60\end{array}$ \\
\hline Output torque max. (Nm) & $2 \times 10,500$ \\
\hline \multicolumn{2}{|l|}{ Battery System } \\
\hline Battery type & Lithium-ion \\
\hline Battery capacity (kWh) & $196(320 \mathrm{Ah})$ \\
\hline Rated voltage (V) & 650 \\
\hline $\begin{array}{l}\text { Max. current discharge } \\
\text { (A) }\end{array}$ & 350 \\
\hline \multicolumn{2}{|l|}{ Performance } \\
\hline Passenger seat & 43 \\
\hline Climbing ability $(\%)$ & 26.8 \\
\hline Max. speed (km/h) & 90 \\
\hline Max. range $(\mathrm{km})$ & $\begin{array}{l}150 \text { (with full load and } \\
\text { air-condition) }\end{array}$ \\
\hline
\end{tabular}

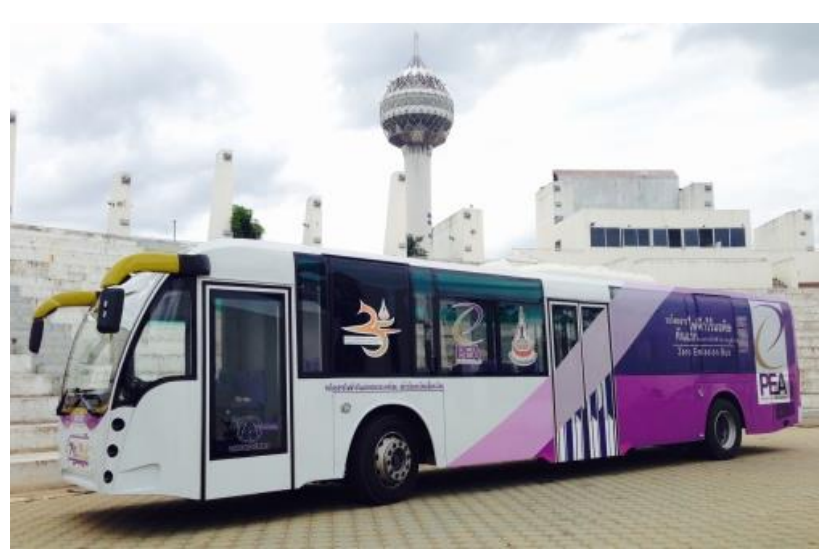

Figure.3 PEA-Zebus running test

emission bus (PEA-Zebus), developed by Suranaree University of Technology (SUT), Thailand is used as a test vehicle as shown in Fig. 3. The specifications of the bus are as given in Table 2 .

\subsection{Field data collection}

The overview of the test and data collection procedure is shown in Fig. 4. The bus was driven for $1,000 \mathrm{~km}$ around the Suranaree University of Technology (SUT) route as shown in Fig. 5 and Data set 1 was collected during the first $143.8 \mathrm{~km}$ test drive and Data set 2 was collected during the last $159.1 \mathrm{~km}$ test drive, both starting with a fully charged battery
(100\% SOC). Data set 3 was collected during a 16.17 $\mathrm{km}$ test drive on an extra-urban route after the bus had been in use (driven) for a certain amount of time. The initial battery's SOC for Data set 3 is to be estimated as aforementioned and then the SOC is to be updated using coulomb counting. The total time and ampshour (Ah) consumption for Data set 3 is 925 seconds and 17.23 Ah, respectively. The energy needed to recharge the battery to be fully charged will be used to validate the initial SOC estimation for Data set 3 . For all the Data sets, they were recorded after every 1 second via CAN (Control Area Network) bus as shown in Fig. 6. A one-second sampling time is considered suitable for the Battery Management System [14].

Three data sets were collected, Data set 1 and Data set 2 comprises of battery terminal voltage, battery discharge current and battery SOC as shown in Fig. 7 and 8 respectively, while Data set 3 comprises of only battery terminal voltage and battery discharge current, while the battery SOC is to be estimated. For Data set 1 and Data set 2, the battery's initial SOC is $100 \%$, and the final SOC is $34.4 \%$ and $28.8 \%$ respectively. The SOC data is updated by Coulomb (amps-hour) Counting.

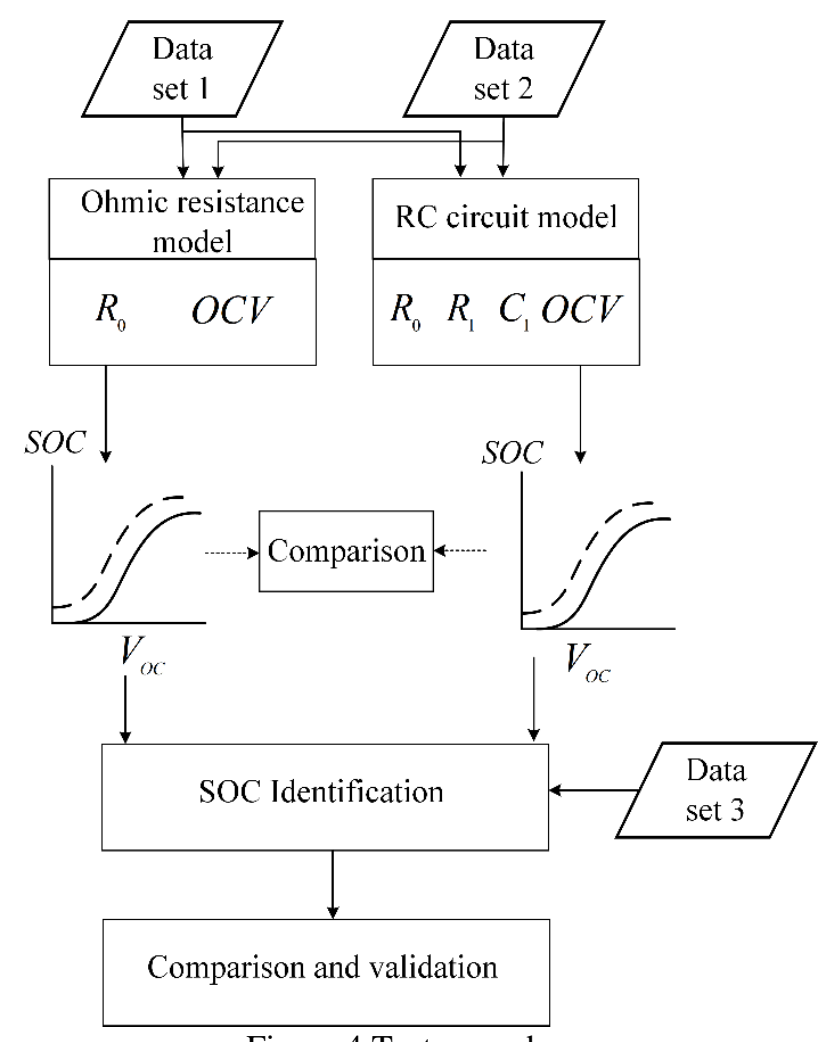

Figure.4 Test procedure 


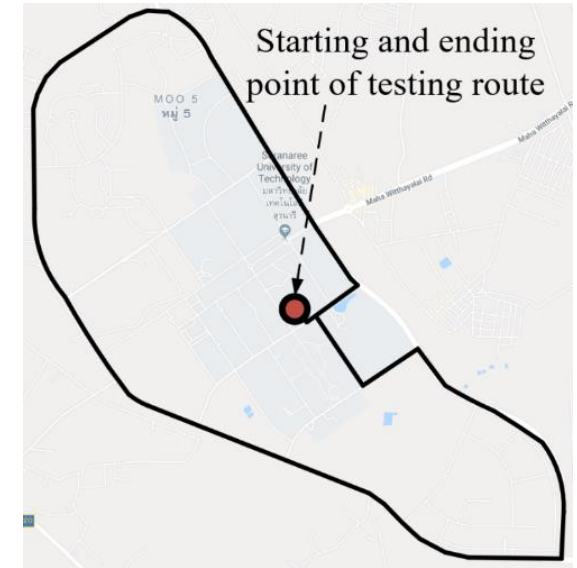

Figure. 5 Test route for Data set 1 and Data set 2

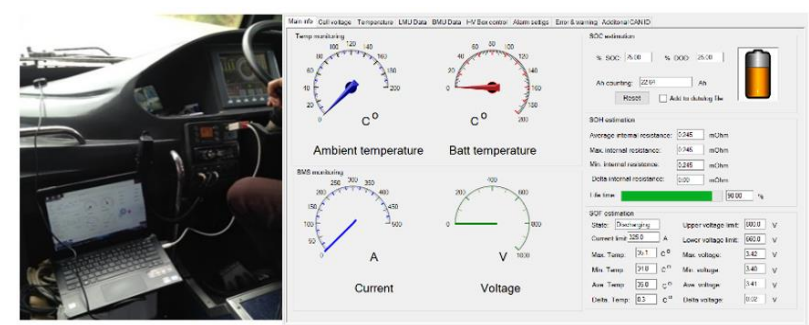

Figure.6 Data measuring and logging via CAN bus

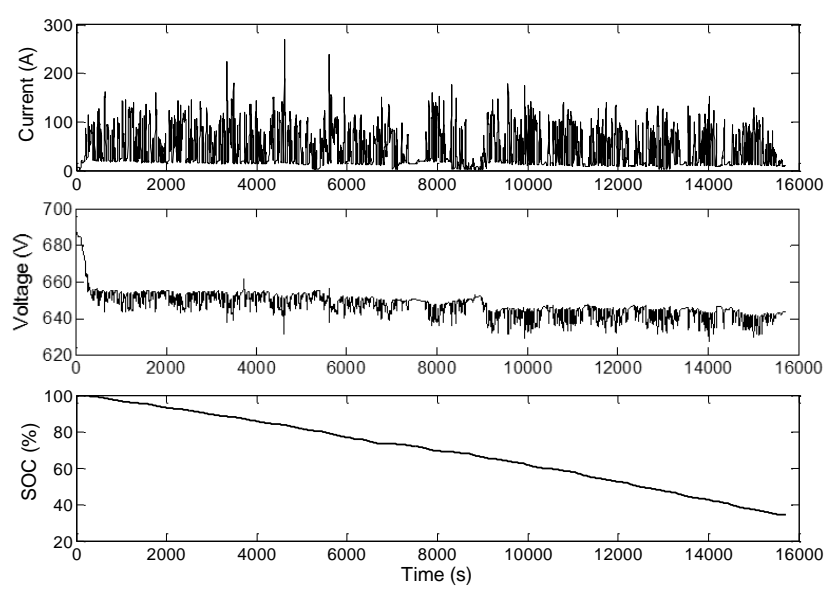

Figure.7 Measured parameters, Data set 1

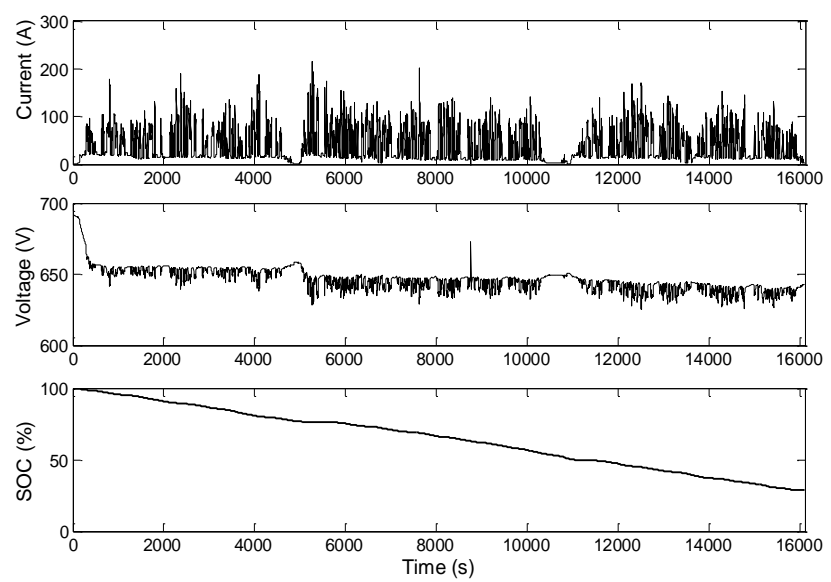

Figure. 8 Measured parameters, Data set 2
Open circuit voltage polynomial curve fitting is done for ohmic resistance model and RC circuit model; The polynomial functions for Data set 1 (ohmic resistance model), Data set 1 (RC circuit model), Data set 2 (ohmic resistance model) and Data set 2 ( $\mathrm{RC}$ circuit model) are presented as shown in Eqs. (10) - (13), respectively. The curves are then used to estimate the initial battery SOC.

$$
\begin{aligned}
& S O C_{\text {Ohm, data } 1}=-1.26 \times 10^{-4}\left(V_{O C}^{4}\right)+0.34\left(V_{O C}^{3}\right)-\cdots \\
& 3.48 \times 10^{2}\left(V_{O C}^{2}\right)+1.57 \times 10^{5}\left(V_{O C}\right)-\cdots \\
& 2.67 \times 10^{7} \\
& S O C_{R C, \text { data } 1}=4.34 \times 10^{-4}\left(V_{O C}^{4}\right)-1.14\left(V_{O C}^{3}\right)-\cdots \\
& 1.13 \times 10^{3}\left(V_{O C}^{2}\right)-4.97 \times 10^{5}\left(V_{O C}\right)-\cdots \\
& 8.91 \times 10^{7} \\
& 3.27 \times 10^{2}\left(V_{O C}^{2}\right)+1.48 \times 10^{5}\left(V_{O C}\right)-\cdots \\
& 2.5 \times 10^{7} \\
& 5.21 \times 10^{3}\left(V_{O C}\right)-1.16 \times 10^{6}
\end{aligned}
$$

\subsection{Proposed SOC estimation algorithm}

The proposed method uses two battery equivalent circuit models; the ohmic resistance equivalent circuit model and RC equivalent circuit model as shown in Fig. 1 and Fig. 2 respectively. The RC equivalent circuit model has higher accuracy than the ohmic resistance equivalent circuit model which can be seen from the estimated battery terminal voltage from the two models compared to the actual measurement. Thus, the RC equivalent circuit model is used to update the SOC-OCV curve while the ohmic resistance equivalent circuit model is used to track changes in battery internal resistance. Data set 1 and Data set 2 are used to identify battery parameters and the SOC-OCV curve. The RLSE algorithm is used online (real-time) with the Ohmic Resistance Model to track the ohmic resistance. The high accuracy RC equivalent circuit model uses the SQP heuristic process to update the SOC-OCV curve offline.

Using the battery current and terminal voltage, the ohmic resistance equivalent circuit model estimates the battery internal resistance and tracks its changes as shown in third stage of Fig. 9, once the change in the estimated internal resistance is significant, a request is sent to the high accuracy RC equivalent circuit to update the SOC-OCV curve as shown in the fourth stage in Fig. 9. The SOC-OCV curve is updated offline using the battery terminal voltage and 


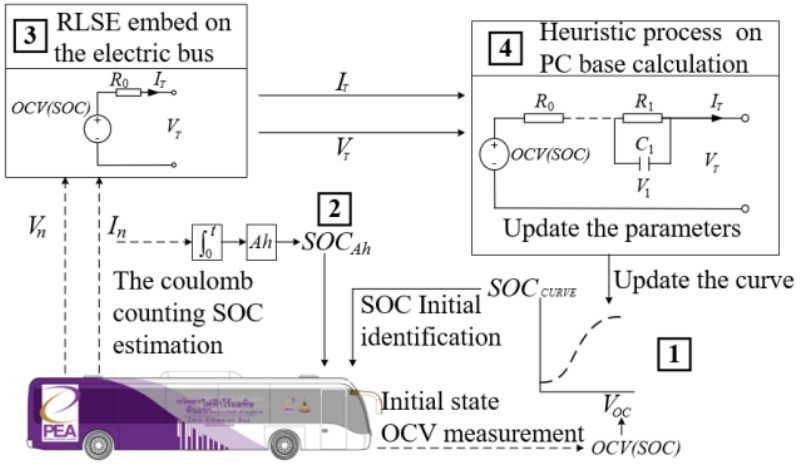

Figure.9 The overview of proposed algorithm

current data of the latest discharging cycle. The upto-date SOC-OCV curve is used to estimate the battery's initial SOC as shown in the first stage of Fig. 9.

First, before any load is connected to the traction battery, the traction battery SOC is estimated by a directly measured voltage from the battery terminals using the SOC-OCV curve as shown in first stage. Since no load is connected beforehand, the initial measured voltage is the OCV. Using the SOC-OCV relationship in the primary stage, this eliminates the need to add for a compensated term for a selfdischarge effect in the battery model. Therefore, the unknown parameters of the model are reduced. At the next stage in the algorithm processes, After the load is connected. The coulomb counting method is used to update the battery SOC by continuously integrating battery discharge currents dictated by the bus's intermittent load as shown in the second stage. The final SOC coulomb counting is then stored and estimated, where the initial battery SOC is validated by energy, needed to fully charge the battery. After that, using the battery current and terminal voltage, the ohmic resistance equivalent circuit model estimates the battery internal resistance and tracks its changes as shown in third stage, once the change in the estimated internal resistance is significant, a request is sent to the high accuracy RC equivalent circuit to update the SOC-OCV curve as shown in the fourth stage. Finally, The SOC-OCV curve is updated offline using the battery terminal voltage and current data of the latest discharging cycle. The upto-date SOC-OCV curve is used to estimate the battery's initial SOC as shown in the first stage.

\section{Results and discussion}

The comparison between the estimated battery pack terminal voltage from two battery models (ohmic resistance model and RC model) and the actual battery pack voltage is presented in this section. This is done to demonstrate the accuracy of the RC battery model. The estimated battery pack SOC uses the proposed method which has been presented and validated.

\subsection{Battery models comparison}

Battery pack terminal voltage from the two battery models are compared with the actual field measured voltage in Fig. 10, Fig. 11, and Fig. 12 using Data set 1, Data set 2, and Data set 3, respectively. All figures illustrate the same pattern; the battery terminal voltage decreases slightly with time. However, as it can be seen clearly, the estimated battery terminal voltage from the ohmic resistance model does not match with the measured voltage especially at the beginning of the test and when the voltage suddenly changes. In contrast, the estimated battery terminal voltage from the RC equivalent circuit model matches with the measured voltage throughout the test period. As presented in Table 3, the mean square error (MSE) from all RC equivalent circuit model is smaller than that of the ohmic resistance equivalent circuit model. Therefore, the RC equivalent circuit model has a higher accuracy than the ohmic resistance model and this is the reason it was used to update SOC-OCV solution as aforementioned.

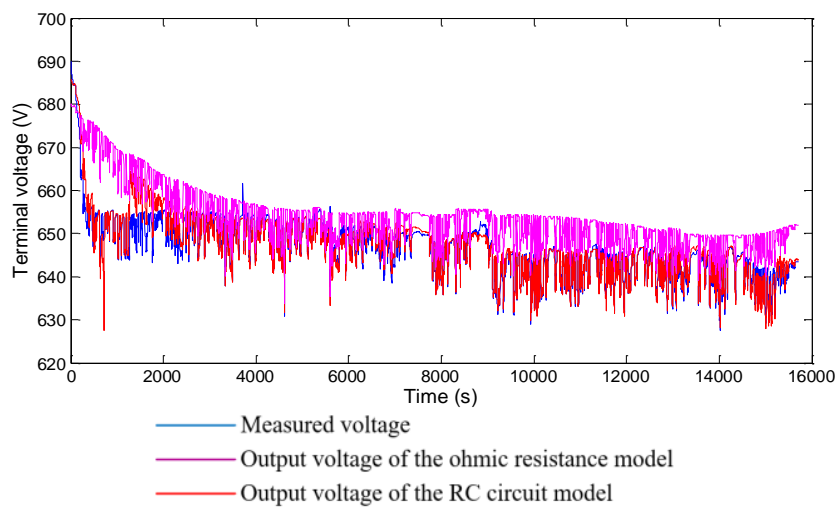

Figure.10 Battery terminal voltage comparison from Data set 1

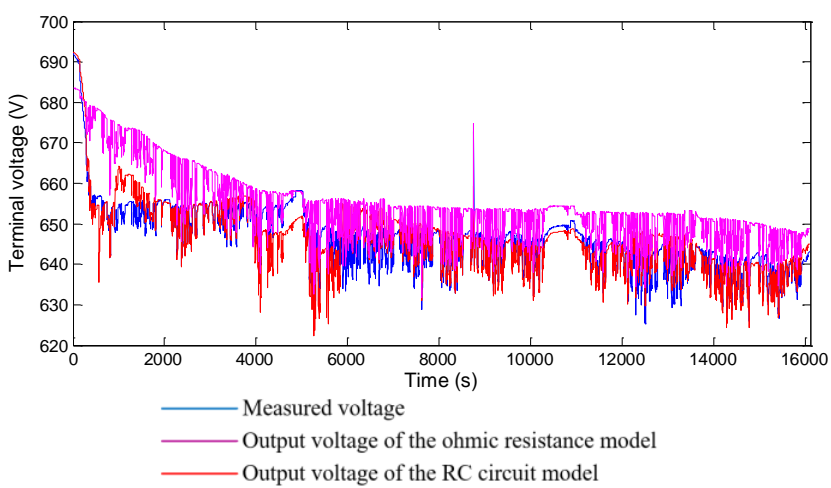

Figure.11 Battery terminal voltage comparison from Data set 2 


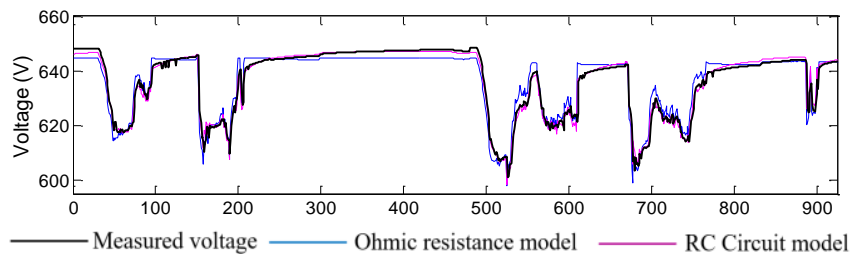

Figure.12 Battery terminal voltage comparison from Data set 3

Table 3. Estimated parameters for all data sets

\begin{tabular}{|c|c|c|c|c|c|c|}
\hline \multirow{2}{*}{$\begin{array}{c}\text { Data } \\
\text { set }\end{array}$} & \multirow{2}{*}{ Model } & \multicolumn{4}{|c|}{ Estimated parameter } & \multirow{2}{*}{ MSE } \\
\hline & & $R_{o}$ & OCV & $R_{I}$ & $C_{l}$ & \\
\hline \multirow{2}{*}{1} & ohmic & 0.09 & 650.63 & - & - & 64.07 \\
\hline & $\mathrm{RC}$ & 0.08 & 644.57 & 0.069 & 149.83 & 10.13 \\
\hline \multirow{2}{*}{2} & ohmic & 0.12 & 650.90 & - & - & 83.26 \\
\hline & $\mathrm{RC}$ & 0.08 & 642.75 & 0.068 & 149.69 & 12.60 \\
\hline \multirow{2}{*}{3} & ohmic & 0.13 & 645.09 & - & - & 11.62 \\
\hline & $\mathrm{RC}$ & 0.10 & 647.34 & 0.059 & 438.61 & 03.73 \\
\hline
\end{tabular}

\subsection{SOC estimation and validation}

From Data set 3 in Fig. 13, The voltage and current presented, are measured directly. The initial SOC is estimated using SOC-OCV curve and then the SOC is updated using coulomb counting as presented. During the current (discharge current) inrush, due to the vehicle acceleration or hill climbing, the battery's terminal voltage falls dramatically and the Ampshour (Ah) count increases considerably. When the current drops sharply to a steady low current state, the voltage rapidly rises and then gradually increases with hysteresis voltage, the Ah count slightly increases.

Fig. 14 shows the estimated battery parameters using ohmic resistance model and RC model. Similarly to Data set 1 and Data set 2, the estimated $R_{o}$ using ohmic resistance model has minimal fluctuation at the beginning and decreases to a stable value which is around 0.127 Ohms. The RC equivalent circuit model gives only one ohmic resistance which is $0.101 \mathrm{Ohms}\left(R_{l}\right.$ and $C_{l}$ of the RC equivalent circuit model are shown in the Table 3$)$. The OCV from both models are fairly constant.

As mentioned previously, the initial SOC is estimated using directly initial measured battery terminal voltage (no loads voltage) and the SOCOCV curve. From Data set 3, the initial no load voltage (open circuit voltage) was $648.08 \mathrm{~V}$. This voltage is substituted in the up-to-date polynomial curve fitting to estimate the initial SOC, and in this case the initial SOC was $56.70 \%$. Knowing the battery capacity $(320 \mathrm{Ah})$ and having established the initial SOC, coulomb method is used to update the SOC, in this case the final SOC was $51.31 \%$ as presented in Table 4 (The RC model of Data 2 or the

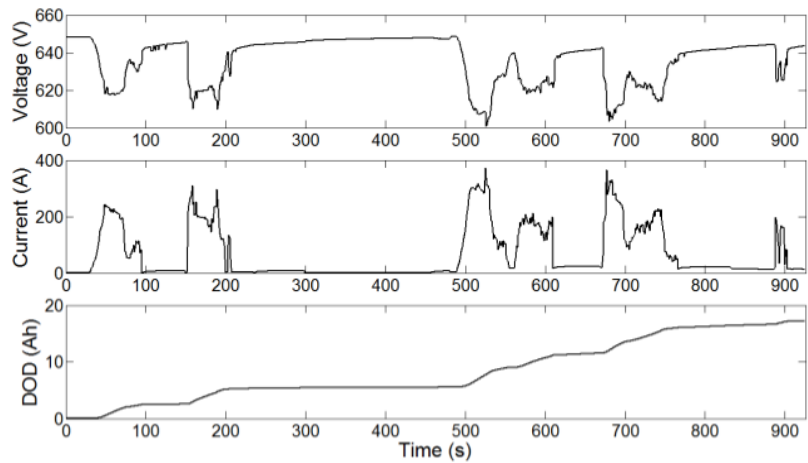

Figure.13 Data set 3 from proposed algorithm
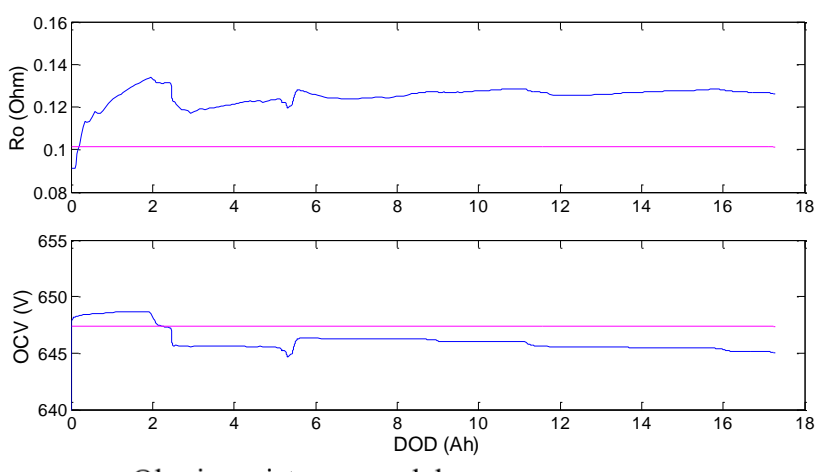

Ohmic resistance model RC Circuit model

Figure.14 Battery pack estimated parameters from Data set 3

Table 4. Estimated SOC

\begin{tabular}{|c|l|c|c|c|}
\hline $\begin{array}{c}\text { Data } \\
\text { set }\end{array}$ & Model & $\begin{array}{c}\text { Estimated } \\
\text { initial SOC } \\
\text { using Data } \\
\text { set 3 (\%) }\end{array}$ & $\begin{array}{c}\text { Estimated } \\
\text { final SOC } \\
\text { using Data } \\
\text { set 3 (\%) }\end{array}$ & $\begin{array}{c}\text { Error } \\
(\%)\end{array}$ \\
\hline \multirow{2}{*}{1} & Ohmic & 3.43 & N/A & N/A \\
\cline { 2 - 5 } & RC & 50.65 & 45.26 & 9.36 \\
\hline \multirow{2}{*}{2} & Ohmic & N/A & N/A & N/A \\
\cline { 2 - 5 } & $\begin{array}{l}\text { Purposed } \\
\text { algorithm }\end{array}$ & 56.70 & 51.31 & 3.31 \\
\hline
\end{tabular}

proposed algorithm), the coulomb count was 17.23 Ah. The Amps hours needed to fully charge the battery pack is used to validate the final estimated battery SOC, thus indirectly validating the initial estimated SOC. In this case the Ah needed to fully charge the battery pack was 145.2Ah (recorded from battery charger), the estimation of SOC error is also presented in Table 4.

In order to comprehend the estimation of SOC, if Data set 1 and the ohmic resistance model were to be used to create a SOC-OCV curve and estimate an initial battery pack SOC, whereas Table 4 also presents the estimated SOC and percentage error using Data set 1, and ohmic resistance model. By using Data set 1 and ohmic resistance model, the initial battery pack SOC (3.43 \%) is estimated by using Eq. (10), resulting in the final SOC as negative considered as an invalid result. For Data set 2, the 
initial SOC from the ohmic resistance model is negative, estimated using Eq. (12). Thus, both the initial and final SOC are invalid. Using Data set 1 and RC model, initial SOC is estimated using Eq. (11). The initial SOC, final SOC and mean square error are $50.65 \%, 45.26 \%$ and $9.36 \%$, respectively. The error is higher than that from Data set 2.

\section{Conclusion}

An accurate battery pack state of charge (SOC) estimation is crucial for an electric vehicle as it reflects how long a vehicle can travel before recharging. Battery parameters change as a battery degrades and a battery model is required to take the changes into account for accuracy throughout battery lifetime. Such model is computational intensive, with limited processing performance hardware found in vehicles. A battery model's fidelity is usually compromised. This paper provides research on the use of two battery models in order to accurately estimate traction battery SOC. The ohmic resistance model is used to track changes in battery internal resistance, when the change is significant a request is sent to update battery SOC-OCV curve using resistor-capacitor (RC) model. The up-to-date SOCOCV curve is used to estimate battery initial SOC. Hence, coulomb counting is used to update the SOC. Using the provided field data from the PEA Ze-Bus (Zero-Emission bus of the Provincial Electricity Authority of Thailand) In conclusion, the proposed model is validated, since the SOC estimation error was less significant (3.31\%). It is also evident that the RC model has higher accuracy than the ohmic resistance model, and latest Data set provides a more accurate SOC-OCV curve than the prior data. Hence, resulting in the reason the proposed algorithm can be applied to update the SOC-OCV curve to reduce the state of charge estimation error.

For future research, the proposed algorithm will implement on embedded hardware in the electric bus. After that, further research will also focus on investigating the difference between the highly intermittent load of the urban route and using the highly constant velocity of the extra-urban route that effects on battery pack capacity and SOC identification.

\section{References}

[1] J. Shen, S. Dusmez, and A. Khaligh, "An advanced electro-thermal cycle-lifetime estimation model for LiFePO4 batteries", In: Proc. of IEEE Transportation Electrification Conference and Expo, pp.1-6, 2013.
[2] Z. Zhang, "Modeling a lithium-ion battery based on a threshold model", In: Proc. of Int. Conf. Advanced Mechatronic Systems, pp.301-305, 2015.

[3] H. Miyamoto, M. Morimoto, and K. Morita, "On-line SOC Estimation of Battery for Wireless Tram Car. In: Proc. of 7th Int. Conf. Power Electronics and Drive Systems, pp.16241627, 2007.

[4] W. Saikong and T. Kulworawanichpong, "Comparative Study of Energy Consumption for Electric Vehicles with Various On-board Energy Storage Systems", Energy Procedia, Vol.138, pp.81-86, 2017.

[5] A. Ramamurthy, S. Notani, and S. Bhattacharya, "Advanced lithium ion battery modeling and power stage integration technique", In: Proc. of IEEE Energy Conversion Congress and Exposition, pp.1485-1492, 2010.

[6] T. Huria, M. Ceraolo, J. Gazzarri, and R. Jackey, "High fidelity electrical model with thermal dependence for characterization and simulation of high power lithium battery cells", In: Proc. of IEEE International Electric Vehicle Conference, pp.1-8, 2012.

[7] R. Jackey, M. Saginaw, P. Sanghvi, J. Gazzarri, T. Huria, and M. Ceraolo, Battery Model Parameter Estimation Using a Layered Technique: An Example Using a Lithium Iron Phosphate Cell, SAE Technical Paper 2013-011547, 2013.

[8] D. Danilov and PHL. Notten, "Adaptive Battery Management Systems for the new generation of Electrical Vehicles", In: Proc. of IEEE Vehicle Power and Propulsion Conference, pp.317-320, 2009.

[9] DD. Patel, S. Sharma, and ZM. Salameh, "Electric vehicle grade lithium polymer battery model using PSCAD", In: Proc. of IEEE PES General Meeting Conference and Exposition, pp.1-5, 2014.

[10] B. Xu, A. Oudalov, A. Ulbig, G. Andersson, and D. Kirschen, "Modeling of Lithium-Ion Battery Degradation for Cell Life Assessment", IEEE Transactions on Smart Grid, Vol.9, No.2, pp.1131-1140, 2018.

[11] J. Gazzarri, N. Shrivastava, R. Jackey, and C. Borghesani, "Battery Pack Modeling, Simulation, and Deployment on a Multicore Real Time Target", SAE International Journal of Aerospace, Vol.7, No.2, pp.207-213, 2014.

[12] J. Wu, C, Zhang, and Z. Chen, "A novel lithiumion battery model for state of charge estimation under dynamic currents", In: Proc. of 4th Int. Conf. Electric Power and Energy Conversion 
Systems, Sharjah, United Arab Emirates, pp.1-6, 2015.

[13] S. Zechang, W. Xuezhe, and D. Haifeng, "Battery management systems in the Chinamade "Start" series FCHVs", In: Proc. of IEEE Vehicle Power and Propulsion Conference, pp.1-6, 2008.

[14] D. Andrea, Battery management systems for large lithium-ion battery packs. Artech house Norwood, MA, 2010.

[15] WY. Yang, WW. Cao, TS. Chung, and J. Morris, Applied numerical methods using MATLAB, John Wiley \& Sons Inc. Publication, New Jersey, 2015.

[16] EKP. Ching and SH. Zak, An introduction to optimization second edition, John Wiley \& Sons Inc. Publication, Canada, 2001.

[17] J. Jaguemont, L. Boulon, and Y. Dubé, "Characterization and Modeling of a HybridElectric-Vehicle Lithium-Ion Battery Pack at Low Temperatures", IEEE Transactions on Vehicular Technology, Vol.65, No.1, pp.1-14, 2016.

[18] N. Watrin, R. Roche, H. Ostermann, B. Blunier, and A. Miraoui, "Multiphysical Lithium-Based Battery Model for Use in State-of-Charge Determination", IEEE Transactions on Vehicular Technology, Vol.61, No.8, pp.34203429, 2012.

[19] T. Huria, M. Ceraolo, J. Gazzarri, and R. Jackey, Simplified Extended Kalman Filter Observer for SOC Estimation of Commercial Power-Oriented LFP Lithium Battery Cells, SAE Technical Paper 2013-01-1544, 2013.

[20] H. Rahimi-Eichi, B. Balagopal, MY. Chow, and TJ. Yeo, "Sensitivity analysis of lithium-ion battery model to battery parameters", In: Proc. of 39th Annual Conf. IEEE Industrial Electronics Society, pp.6794-6799, 2013. 